\title{
Sistematización de la atención de enfermería en pacientes con insuficiencia cardiaca etapa IV
}

\section{Systematization of nursing care in patients with heart failure stage IV \\ Sistematização da assistência de enfermagem em paciente com insuficiência cardíaca estágio IV}

Jéssica Naiara de Medeiros Araújo ${ }^{1}$, Maria Isabel da Conceição Dias Fernandes ${ }^{2}$, Andréa Tayse de Lima Gomes ${ }^{3}$,

Millena Freire Delgado ${ }^{4}$, Ana Luisa Brandão de Carvalho Lira ${ }^{5}$, Allyne Fortes Vitor ${ }^{6}$.

${ }^{1,3,4}$ Enfermeira. Mestranda do programa de pós-graduação em enfermagem da Universidade Federal do Rio Grande do Norte (UFRN).

${ }^{2}$ Enfermeira. Doutoranda do programa de pós-graduação em enfermagem da Universidade Federal do Rio Grande do Norte (UFRN).

${ }^{5,6}$ Enfermeira. Doutora em enfermagem. Professora Adjunta II do Departamento de Enfermagem da Universidade Federal do Rio Grande do Norte (UFRN).

Cómo citar este artículo en edición digital: Araujo, J.N.M., Fernandes, M.I.C.D., Gomes, A.T.L., Delgado, M. F., Lira, A.L.B.C., Vitor, A.F. (2017). Sistematización de la atención de enfermería en pacientes con insuficiencia cardiaca etapa IV.

Cultura de los Cuidados (Edición digital), 21(48). Recuperado de < http://dx.doi.org/10.14198/cuid.2017.48.25>

Correspondencia: Jéssica Naiara de Medeiros Araújo. Rua Dom Joaquim de Almeida, 2076, apto 202/ Lagoa Nova, CEP: 59056-140, Natal/RN/Brasil.

Correo electrónico: jessicanaiara_rn@hotmail.com

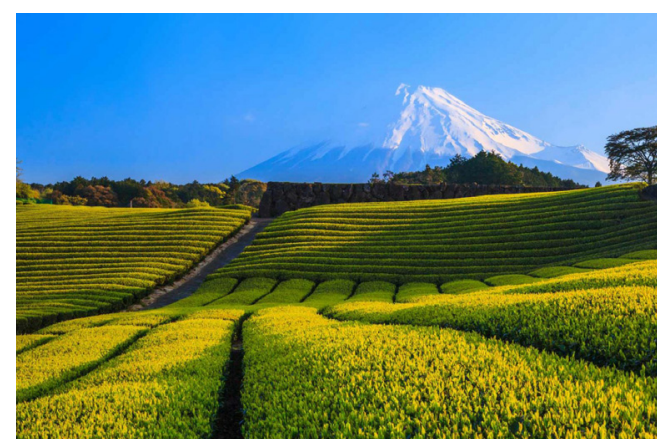

\section{ABSTRACT}

This is a case study, with the objective of to describe the application of the nursing process in a patient with stage IV heart failure. Conducted in a university hospital located in Northeastern Brazil. Data were collected in the month October 2012, using a structured interview and physical examination. The diagnoses identified were: Impaired skin integrity, Deprivation of sleep, Constipation and excessive fluid volume. Was traced the result - Wound healing: second intention, and applied to the nursing intervention - Care with pressure ulcers. Thus, through the implementation of the nursing care subsidized by the systematization of nursing care, it was observed that the desired results were achieved with expert.

Key words: Nursing care, heart failure, pressure ulcer, nursing.

\section{RESUMEN}

Se trata de un estudio de caso, con el objetivo de describir la aplicación del proceso de enfermería en un paciente con insuficiencia cardiaca en etapa IV. Realizado en un hospital universitario situado en el Nordeste del Brasil. Los datos fueron recolectados en el mes octubre 2012 a través de un guión de entrevista y examen físico. Se identificaron los diagnósticos: Integridad de la piel alterada, La privación del sueño, Constipación y Volumen excesivo de líquidos. Se elaboró el resultado - Cicatrización de heridas: segunda intención, y se aplicó la intervención de enfermería - Cuidado de las úlceras por presión. 
De este modo, a través de la implementación de la atención de enfermería subsidiada por la sistematización de la asistencia de enfermería, se observó que los resultados deseados se lograron con experto.

Palabras clave: Atención de enfermería, insuficiencia cardíaca, úlcera por Presión, enfermería.

\section{RESUMO}

Trata-se de um estudo de caso, com o objetivo de descrever a aplicação do processo de enfermagem em um paciente com insuficiência cardíaca estágio IV. Realizado em um hospital universitário localizado no Nordeste do Brasil. Os dados foram coletados no mês outubro de 2012, por meio de um roteiro de entrevista e de exame físico. Os diagnósticos identificados foram: Integridade da pele prejudicada, Privação de sono, Constipação e Volume de líquidos excessivo. Foi traçado o resultado - Cicatrização de feridas: segunda intenção, e aplicou-se a intervenção de enfermagem - Cuidados com úlceras de pressão. Assim, por meio da implementação dos cuidados de enfermagem subsidiados pela sistematização da assistência de enfermagem, observou-se que os resultados almejados foram alcançados com exímio.

Palavras-chave: Cuidados de enfermagem, insuficiência cardíaca, úlcera por pressão, enfermagem.

\section{INTRODUÇÃO}

A insuficiência cardíaca (IC) é uma síndrome crônica caracterizada por falência cardíaca e incapacidade de bombear o suprimento de sangue necessário para satisfazer as demandas metabólicas dos tecidos (Nogueira, Rassi, \& Corrêa, 2010).
No Brasil, existe cerca de dois milhões de pessoas com IC, sendo 240 mil novos casos diagnosticados por ano. Em 2009, foi causa mais frequente de internação em idosos, população na qual a prevalência da doença e o índice de mortalidade é bastante elevado (Santos, Santo, Pestana, Daher, \& Santana, 2011; Gaui, Klein, \& Oliveira, 2010).

Apresenta como principais sinais e sintomas a dispneia e a fadiga durante o exercício ou Atividade de Vida Diária (AVD). Tais indícios clínicos são decorrentes da função pulmonar diminuída, em virtude do bombeamento ineficaz de oxigênio e nutrientes pelo coração, promovendo a intolerância ao exercício (Lima et al., 2011; Naso et al., 2011).

A New York Heart Association (NYHA) classifica a IC nos escores de I a IV, os quais são estipulados em consonância com os sintomas relatados pelo paciente (Naso et al., 2011). No início, o comprometimento ocasionado pela diminuição do débito cardíaco se manifesta durante o exercício, e com a progressão da doença, tal comprometimento é observado até mesmo no repouso (Bocchi et al., 2009).

Desse modo, pacientes classificados nos níveis III e IV, possuem dificuldade em realizar grandes esforços ou atividades da vida diária, tornando-se mais dependentes. Tal assertiva corrobora com um estudo envolvendo pacientes com mais de 60 anos de idade, o qual indicou como causa comum de internação, às doenças cardiovasculares, e dentre os diagnósticos de enfermagem prevalentes, o déficit no autocuidado, atrelado a alterações na nutrição e risco de infecção (Franzen et al., 2007).

Diante do exposto, pacientes idosos hospitalizados, acometidos pela IC grau IV, possui um risco acentuado para desenvolver úlceras por pressão em virtude da incapacidade gerada pela doença e por fatores intrínsecos da idade 
(Fernandes et al., 2012). Portanto, a assistência de enfermagem deve contribuir, sobremaneira, na prevenção e tratamento dos pacientes com úlcera por pressão. Para isso, estes indivíduos deverão ser assistidos de modo sistemático e organizado.

Destarte, a utilização da Sistematização da Assistência de Enfermagem (SAE) é fundamental, pois essa organiza o trabalho de enfermagem quanto ao método, instrumentos e pessoal (Luiz, Padoin, Neves, Ribeiro, \& Tronco, 2010). Direcionando as atividades da equipe de enfermagem, permitindo a identificação das necessidades do paciente, demonstrando uma proposta para o atendimento e o cuidado (Menezes, Priel, \& Pereira, 2011). A SAE é operacionalizada por meio do Processo de Enfermagem (PE), o qual é um método científico que proporciona a organização do trabalho de enfermagem, sendo constituído por cinco etapas que se inter-relacionam, quais seja: coleta de dados, diagnósticos de enfermagem, planejamento, implementação e avaliação (Luiz, Padoin, Neves, Ribeiro, \& Tronco, 2010). A partir do exposto, este trabalho objetiva descrever a experiência da aplicação do processo de enfermagem em um paciente com insuficiência cardíaca estágio IV.

\section{MARCO TEÓRICO}

Wanda de Aguiar Horta, primeira enfermeira brasileira a falar de teoria no âmbito profissional, embasou-se nos conceitos da motivação Humana de Abraham Maslow para elaborar a Teoria das Necessidades Humanas Básicas. Assim, propôs a assistência de enfermagem sistematizada, introduzindo uma nova visão de cuidar no Brasil (Tannure, \& Gonçalves, 2008). As necessidades humanas básicas são definidas como "estados de tensões, conscientes ou não, resultantes de desequilíbrio hemodinâmico dos fenômenos vitais". Além disso, Horta ressalta que todas as necessidades estão inter-relacionadas e fazem parte de um todo, o ser humano (Horta, 1979).

Embora a Teoria das Necessidades Humanas Básicas tenha sido fundamentada na proposta de Maslow, na enfermagem, a preferência é utilizar a denominação estabelecida por João Mohana, a qual consiste em: necessidades de nível psicobiológica, psicossocial e psicoespiritual (Horta, 1979). A aplicação da Teoria das Necessidades Humanas Básicas permite que a enfermeira atenda ao ser humano com ações de enfermagem planejadas e embasadas na sua percepção e análise de comportamento humano, frente a uma situação ou condição específica (Gonçalves, 2008). Desse modo, o modelo conceitual de Wanda Horta, conforme as determinadas subcategorias de sua abordagem teórica permite a associação com os pontos chaves da SAE e do PE, uma vez que busca prestar assistência integral ao indivíduo embasada na identificação das necessidades humanas básicas durante processo saúde-doença.

Nesse sentido a SAE configura-se como uma metodologia para organizar e realizar o cuidado fundamentado nos princípios do método científico. No entanto, consiste em uma atividade privativa no processo de trabalho do enfermeiro, a qual possibilita o desenvolvimento de ações capazes de modificar o estado do processo de vida e de saúde-doença dos indivíduos (COFEN, 2002; Truppel, Meier, Calixto, Peruzzo, \& Crozeta, 2009).

Além disso, a SAE subsidia a prática da enfermagem que contribuem veementemente para a promoção, prevenção, recuperação e reabilitação da saúde do indivíduo, família e comunidade (COFEN, 2002). Nesse ínterim, a SAE presume a organização em um sistema que requer um conjunto de elementos, dinami- 
camente inter-relacionados, denominado $\mathrm{PE}$ (Carvalho, \& Bachion, 2009), o qual é constituído basicamente por cinco etapas, a saber: 1) Histórico de Enfermagem - coleta de dados e exame físico; 2) Diagnóstico de Enfermagem - pautado nos problemas anteriormente identificados; 3) Planejamento de Enfermagem; 4) Implementação de Enfermagem; e, 5) Avaliação de Enfermagem (COFEN, 2009). Tal processo representa o instrumento de trabalho do enfermeiro, cujo objetivo é auxiliar na identificação das necessidades do paciente, apresentando uma proposta ao seu atendimento e cuidado, de forma a direcionar a equipe de enfermagem nas ações a serem realizadas (COFEN, 2009).

\section{METODOLOGIA}

Trata-se de um estudo de caso sobre uma paciente com insuficiência cardíaca, classificada no estágio IV, o qual foi realizado em uma enfermaria cardiológica de um Hospital Universitário localizado no Nordeste do Brasil. A paciente foi escolhida por conveniência e os dados foram coletados no mês outubro de 2012, por meio de um roteiro de entrevista estruturado baseado na taxonomia II da NANDA Internacional (Herdman, 2013). Além de um roteiro de exame físico com dados do exame físico geral e dos seguimentos do corpo.

Para o desenvolvimento do estudo, foram aplicadas todas as etapas do processo de enfermagem. A coleta iniciou-se por meio da anamnese e do exame físico, sendo posteriormente, traçados os diagnósticos de enfermagem, utilizando-se as etapas do raciocínio clínico e julgamento diagnóstico preconizados na literatura (Risner, 1986). Após o estabelecimento dos diagnósticos, foram traçados os resultados de enfermagem esperados segundo a NOC (Moorhead, Johnson, Maas, \& Swanson, 2010) e as intervenções segundo a NIC (Dochterman,
Bulechek, \& Butcher, 2010) de acordo com o problema identificado. Após o planejamento, as intervenções foram implementadas para o diagnóstico de enfermagem prioritário, e os resultados avaliados.

O projeto foi aprovado pelo Comitê de Ética da Universidade Federal do Rio Grande do Norte sob o parecer 121. 028. Destaca-se que a paciente no estudo foi convidada a participar, com posterior assinatura do termo de consentimento livre e esclarecido.

\section{RESULTADOS}

\section{Apresentação do caso clínico}

J. A. R., 73 anos, sexo feminino, analfabeta, ex-agricultora e atualmente aposentada. Diagnóstico médico de ICC estágio IV, hipertensão arterial sistêmica e hipotireoidismo. Internou-se na instituição hospitalar devido à mixedema, dispneia em repouso e dor abdominal, membros superiores e inferiores. Ao exame físico geral, apresentou estado geral regular, consciente e orientada, pele hipocorada, integridade da pele comprometida por presença de úlcera por pressão sacral grau II, mixedema em abdome e em membros superiores e inferiores $4+/ 4+$. Fácies atípica, normolínea, deambulando com auxílio.

Ao exame físico dos seguimentos, acuidade visual diminuída, pupilas isocóricas e fotoreagentes. Arcada dentária incompleta e língua saburrosa. Acuidade auditiva preservada. Carótidas com batimentos perceptíveis, fortes $\mathrm{e}$ simétricos e presença de estase jugular à $45^{\circ}$. Tórax simétrico, à ausculta pulmonar, estertores finos em ápice direito. $\mathrm{Na}$ inspeção cardíaca, ictus cordis visível e palpável com duas polpas digitais, à ausculta, bulhas normofonéticas em dois tempos. Abdome globoso, resistente à palpação por presença de edema subcutâneo e ruídos hidroaéreos presentes. Membros supe- 
riores e inferiores com musculatura hipotrófica e pulsos periféricos de difícil palpação devido ao edema.

Durante a internação, foi realizado eletrocardiograma, o qual demonstrou bradicardia sinusal e bloqueio atrioventricular de primeiro grau. Na ecocardiografia transtorácica identificou-se aumento do átrio esquerdo e direito; aumento dos diâmetros do ventrículo esquerdo; acinesia com fibrose da parede inferior; refluxo mitral e tricúspide leve.

\section{Aplicação do processo de enfermagem}

De acordo com os sintomas evidenciados na paciente foram elencados quatro Diagnós- ticos de Enfermagem (DE), a saber: Integridade da pele prejudicada; Privação de sono; Constipação e Volume de líquidos excessivo. Dentre os diagnósticos de enfermagem acima expostos, escolheu-se como prioritário, o diagnóstico Integridade da pele prejudicada, tendo-se em vista que o rompimento da superfície da pele conferia à paciente um maior risco de desenvolver infecção, e causava-lhe dor, problema responsável pela privação do sono. Assim, para desenvolver o plano de cuidados direcionado a este diagnóstico de enfermagem específico foram utilizados os sistemas de classificação NIC e NOC, conforme o Quadro 1.

Quadro 1

Plano de cuidados para o diagnóstico de enfermagem prioritário, Integridade da pele prejudicada, baseado nos sistemas de classificação NANDA Internacional, NIC e NOC. Natal/RN, 2015.

\begin{tabular}{|c|c|c|c|c|c|c|c|}
\hline & & Domínio: Se & guranç & a/prote & ção & & \\
\hline $\begin{array}{l}\text { DE: Integridade } \\
\text { rompimento da s } \\
\text { proeminências ós }\end{array}$ & $\begin{array}{l}\text { da pele pi } \\
\text { uperfície d } \\
\text { eas. }\end{array}$ & $\begin{array}{l}\text { rejudicada ev } \\
\text { la pele relaci }\end{array}$ & $\begin{array}{l}\text { idenciad } \\
\text { onada à }\end{array}$ & $\begin{array}{l}\text { a por de } \\
\text { imobiliz }\end{array}$ & struição & $\begin{array}{l}\text { de cam } \\
\text { ca, fator }\end{array}$ & $\begin{array}{l}\text { idas da pele e } \\
\text { s mecânicos e }\end{array}$ \\
\hline Resultado - Cic & atrização c & de feridas: Se & sunda in & tenção & & & \\
\hline Indicadores & $\begin{array}{c}\text { Atual } \\
\left(1^{\circ} \text { dia }\right)\end{array}$ & Esperado & $2^{\circ} \mathrm{dia}$ & $3^{\circ} \mathrm{dia}$ & $4^{\circ} \mathrm{dia}$ & $5^{\circ}$ dia & $\begin{array}{c}\text { Alcançado } \\
\left(6^{\circ} \text { dia }\right)\end{array}$ \\
\hline Granulação & 04 & 05 & 03 & 02 & 04 & - & 05 \\
\hline $\begin{array}{ll}\text { Formação de } \\
\text { cicatriz }\end{array}$ & 01 & 03 & 01 & 01 & 03 & - & 04 \\
\hline $\begin{array}{l}\text { Tamanho } \\
\text { reduzido da } \\
\text { ferida }\end{array}$ & 01 & 03 & 01 & 02 & 03 & - & 04 \\
\hline Soma & 06 & 11 & 05 & 05 & 10 & - & 13 \\
\hline Intervenção - & idados cc & om úlceras de & ressão & & & & \\
\hline $\begin{array}{l}\text { Descrever as car } \\
\text { (I-IV), localizaçã } \\
\text { Monitorar cor, te } \\
\text { Manter úmida a } \\
\text { Aplicar curativos } \\
\text { Monitorar o apar } \\
\text { Mudança de decú }\end{array}$ & $\begin{array}{l}\text { icterísticas } \\
\text {, exsudato } \\
\text { mperatura } \\
\text { ilcera para } \\
\text { conforme } \\
\text { ecimento d } \\
\text { bito cada }\end{array}$ & $\begin{array}{l}\text { da úlcera a } \\
\text {, tecido granu } \\
\text {, edema, umic } \\
\text { ajudar a cica } \\
\text { apropriado; } \\
\text { le sinais e sint } \\
\text { uma ou duas }\end{array}$ & $\begin{array}{l}\text { intervalo } \\
\text { lar ou ne } \\
\text { lade e ap } \\
\text { trização } \\
\text { omas de } \\
\text { loras. }\end{array}$ & $\begin{array}{l}\text { S regula } \\
\text { crosado } \\
\text { arência } \\
\text { infecção }\end{array}$ & $\begin{array}{l}\text { res, incl } \\
\text { e epiteli } \\
\text { da pele a }\end{array}$ & $\begin{array}{l}\text { uindo tar } \\
\text { zação; } \\
\text { to redor; } \\
\text { lento; }\end{array}$ & anho, estágio \\
\hline
\end{tabular}

Fonte: Herdman, 2013; Dochterman, Bulechek, \& Butcher., 2010; Moorhead, Johnson, Maas, \& Swanson, 2010. 


\section{DISCUSSÕES}

Integridade da pele prejudicada foi elencada como prioritária, sendo o foco das ações de enfermagem, pois a presença de pele não íntegra em pacientes hospitalizados pode aumentar o risco de infecção, o que pode agravar ainda mais o estado de saúde, principalmente se este paciente for idoso com uma doença crônica. Tal diagnóstico estava presente em razão de a paciente apresentar uma úlcera por pressão sacral, classificada no estágio II, em virtude da presença de solução de continuidade da pele envolvendo camadas como a epiderme e a derme (Potter, \& Perry, 2013).

As úlceras por pressão ocorrem, geralmente, devido a uma compressão aplicada à pele por um período de tempo maior que a pressão fisiológica de fechamento capilar. Essa pressão prolongada impede o fluxo sanguíneo adequado, reduzindo, assim, a nutrição e a oxigenação da região (Giglio, Martins, \& Dyniewicz, 2007). Este tipo de úlcera é geralmente observado em pacientes com alterações do reflexo da dor, como por exemplo, o lesado medular, pacientes debilitados, idosos cronicamente doentes e pacientes com problemas cerebrais (Costa, Sturtz, Costa, Ferreira, \& Barros, 2005).

A ocorrência da úlcera na paciente do estudo justifica-se pelo fato dessa ser idosa e portadora de IC, uma doença crônica que pode levar à debilidade com a sua progressão a estágios avançados, ocasionando ao paciente uma dificuldade na deambulação e alteração na mobilidade, com propensão maior ao desenvolvimento de úlcera por pressão (Fernandes et al., 2012).

Ademais, idosos hospitalizados apresentam alta predisposição ao desenvolvimento de úlceras por pressão, em virtude de possuírem pele frágil, dificuldade na mobilidade, enve- lhecimento dos órgãos, maior ocorrência de doenças crônico-degenerativas e problemas imunológicos (Fernandes et al., 2012).

Um estudo sobre a prevalência dos fatores de risco para ocorrência de úlceras por pressão em idosos hospitalizados constatou que a idade, principalmente a faixa etária entre 73 a 83 anos e as doenças crônico-degenerativos são os principais fatores predisponentes para o desenvolvimento de úlceras por pressão (Fernandes et al., 2012; Moro, Maurici, Valle, Zaclikevis, \& Kleinubing, 2007; Rogenski, \& Santos, 2005). Dentre as doenças crônicas, os estudos apontam para aquelas que comprometem os sistemas cardiovascular e respiratório (Rogenski, \& Santos, 2005). Tais achados corroboram com o perfil da paciente em estudo.

Relativo ao resultado da NOC, estabelecido para o diagnóstico prioritário, elencou-se o resultado Cicatrização de feridas: segunda intenção. Para o alcance desse resultado foi traçado a intervenção da NIC: cuidado com úlceras por pressão, sendo estipuladas as atividades expostas no Quadro 1.

Sabe-se que os cuidados clínicos da equipe médica e de enfermagem, assim como os da fisioterapia, suporte nutricional e psicológico devem estar envolvidos no tratamento e na prevenção da úlcera por pressão. Entretanto, cuidados específicos da enfermagem devem ser realizados com vistas a promover a cicatrização desse tipo de ferida. Assim, deve-se inspecioná-la no sentido de classificar a úlcera em estágios e avaliar sua progressão. Portanto, a observação da localização, dimensão, estadiamento, presença ou sinais locais de infecção, dor, odor, presença de fístulas e características da pele adjacente é importante (Fernandes et al., 2012).

Deve-se ressaltar, também que, a prevenção de futuras úlceras por pressão, bem como 
o agravamento da já existente deve ser colocado em pauta, pois não adiantará o uso de tratamentos medicamentosos se a causa, a pressão, não for debelada. Estudos mostram que o tratamento visa diminuir as áreas de pressão sobre as saliências ósseas por meio da mobilização continuada em intervalos menores que duas horas, utilização de colchões apropriados, evitar também a umidade cutânea constante, os atritos da pele com as superfícies de contato e melhorar o estado nutricional do paciente (Giglio et al., 2007).

Em contraponto ao pensamento supracitado, estudo verificou que as medidas de prevenção não são bem implementadas na prática hospitalar, pois dos 300 pacientes idosos hospitalizados, com risco de desenvolver úlceras por pressão, em 258, as medidas de prevenção não foram utilizadas (Fernandes et al., 2012).

No tocante à aplicação das intervenções de enfermagem à paciente em estudo, e de acordo com a avaliação dos resultados, foi estabelecida no primeiro dia de acompanhamento, a soma inicial de seis pontos. No segundo dia de acompanhamento ocorreu uma piora do indicador granulação, devido ao aparecimento de fibrina em maior quantidade no leito da lesão, o qual passou de quatro para três, os outros indicadores permaneceram estáveis. No terceiro dia, também ocorreu maior proliferação da fibrina no leito da lesão, ocasionando outra piora no indicador granulação, o qual passou de três para dois. O indicador formação de cicatriz continuou estável e o indicador tamanho reduzido da ferida melhorou, devido à redução da lesão, passando de um para dois.

O tecido de granulação está relacionado com a restauração tissular, sendo favorável à cicatrização em condições de normalidade. $\mathrm{O}$ excesso de fibrina pode desenvolver-se na superfície da ferida, ocasionando uma dificul- dade no crescimento do tecido de granulação e consequentemente retardando a cicatrização (Dealey, 2008). Em virtude disso, foram implementadas ações que visavam à diminuição desse excesso de fibrina, para que ocorresse o crescimento do tecido de granulação.

Destarte, no quarto dia de acompanhamento, o indicador granulação melhorou substancialmente e atingiu a pontuação quatro. $\mathrm{O}$ indicador formação de cicatriz também melhorou, o qual passou de um para três e o indicador tamanho reduzido da ferida também obteve evolução, evoluindo de dois para três. No quinto dia, os indicadores não foram avaliados devido a não visualização da lesão, por não ter ocorrido à troca de curativo. No sexto dia, o indicador granulação atingiu o valor esperado cinco, e o indicador formação de cicatriz e tamanho reduzido da ferida obtiveram melhora chegando a quatro. E por fim, no último dia de acompanhamento, visualizou-se a melhora dos indicadores, os quais ultrapassaram a soma esperada.

\section{CONCLUSÃO}

O diagnóstico de enfermagem identificado como prioritário foi Integridade da pele prejudicada. Desse modo, o resultado esperado da NOC foi Cicatrização de feridas: segunda intenção, cuja intervenção da NIC foi: cuidado com úlceras de pressão. Assim, por meio da implementação dos cuidados de enfermagem subsidiados pela sistematização da assistência de enfermagem, observou-se que os resultados almejados foram alcançados com exímio.

Conclui-se, portanto, que a elaboração e execução do plano de cuidados desenvolvido por meio das taxonomias de enfermagem, respaldado pelo processo de enfermagem, contribui positivamente para a melhoria do estado de saúde do paciente. Contribui, também, para o 
profissional de enfermagem, que consegue melhor visualizar o seu trabalho, e para a instituição de saúde, pois diminui os gastos, já que o problema é solucionado com maior rapidez e eficácia. Dessa forma, sugere-se que sejam realizados mais estudos que comprovem a eficácia, eficiência e efetividade da aplicação do processo de enfermagem em instituições de saúde.

\section{REFERÊNCIAS}

- Bocchi, E. A., et al. (2009). III Diretriz brasileira de insuficiência cardíaca crônica. Arquivos Brasileiros de Cardiologia, 93(1), 1-71. Recuperado de http://www. scielo.br/pdf/abc/v93n1s1/abc93_1s1.pdf

- Carvalho, E. C., \& Bachion, M. M. (2009). Processo de enfermagem e sistematização da assistência de enfermagem - intenção de uso por profissionais de enfermagem. Revista Eletrônica de Enfermagem, 11(3), 466.

- Conselho Federal de Enfermagem (COFEN). (2002). Resolução 272/2002. Dispõe sobre a Sistematização da Assistência de Enfermagem - SAE - nas Instituições de Saúde Brasileiras. Recuperado de http://www.cofen. gov.br/resoluo-cofen-2722002-revogada-pela-resoluaocofen-n-3582009_4309.html

- Conselho Federal de Enfermagem (COFEN). (2009). Resolução 358/2009. Dispõe sobre a Sistematização da Assistência de Enfermagem e a implementação do Processo de Enfermagem em ambientes, públicos ou privados, em que ocorre o cuidado profissional de Enfermagem, e dá outras providências. Recuperado de http://www.cofen. gov.br/resoluo-cofen-3582009 4384.html

- Costa, M. P., Sturtz, G., Costa, F. P. P., Ferreira, M. C., \& Barros, T. E. P., Filho. (2005). Epidemiologia e tratamento das úlceras de pressão: experiência de 77 casos. Acta ortopedica brasileira, 13(3), 124-133.

- Dealey, C. (2008). Cuidando de feridas: um guia para enfermeiras. $3^{\text {a }}$ ed. São Paulo: Atheneu.

- Dochterman, J. M., Bulechek, G. M., \& Butcher, H. K. (2010). Classificação das intervenções de enfermagem (NIC). $5^{\mathrm{a}}$ ed. Rio de Janeiro: Elsevier.
- Fernandes, M. G. M., et al. (2012). Risco para úlcera por pressão em idosos hospitalizados: aplicação da escala de Waterlow. Revista enfermagem UERJ, 20(1), 56-60. Recuperado de http://www.facenf.uerj.br/v20n1/ v20n1a10.pdf

- Franzen, E., et al. (2007). Adultos e idosos com doenças crônicas: implicações para o cuidado de enfermagem. Revista do Hospital de Clínicas de Porto Alegre e Faculdade de Medicina da Universidade do Rio Grande do Sul, 27(2), 28-31. Recuperado de http://seer.ufrgs.br/hcpa/article/ view $/ 2045$

- Gaui, E. M., Klein, C. H., \& Oliveira, G. M. (2010). Mortalidade por insuficiência cardíaca: análise ampliada e tendência temporal em três estados do Brasil. Arquivos Brasileiros de Cardiologia, 94(1), 55-61. Recuperado de http://www.scielo.br/pdf/abc/v94n1/10.pdf

- Giglio, M M., Martins, A. P, \& Dyniewicz, A. M. (2007). Análise do grau de dependência e predisposição à úlcera de pressão em pacientes de hospital universitário. Cogitare enfermagem, 12(1), 62-8.

- Gonçalves, L. H. T. (2008). O cuidado da pessoa idosa no seu processo saúde e doença. In: Schawanke, C. H. A., \& Schneider, R. H. (Org.). Atualizações em geriatria e gerontologia: da pesquisa básica a prática clínica. Porto Alegre: Edipucrs, 123-133.

- Herdman, T. H. (2013). Diagnósticos de enfermagem da NANDA: definições e classificação - 2012/2014. Porto Alegre (RS): Artmed.

- Horta, W. A. (1979). Processos de enfermagem. São Paulo: EPU.

- Lima, E. S., et al. (2011). Effect of ventilatory support on functional capacity in patients with heart failure: a pilot study. Arquivos Brasileiros de Cardiologia, 96(3), 227-232. - Luiz, F. F., Padoin, S. M. M., Neves, E. T., Ribeiro, A. C., \& Tronco, C. S. (2010). A sistematização da assistência de enfermagem na perspectiva da equipe de um hospital de ensino. Revista eletrônica de enfermagem, 12(4), 655-659.

- Menezes, S. R. T., Priel, M. R., \& Pereira, L. L. (2011). Autonomia e vulnerabilidade do enfermeiro na prática da Sistematização da Assistência de Enfermagem. Revista da Escola de Enfermagem da USP, 45(4), 953-958. Recuperado de http://www.scielo.br/pdf/reeusp/v45n4/ 


\section{Cultura de las Cuidados}

v45n4a23.pdf

- Moorhead, S., Johnson, M., Maas, M. L., \& Swanson, E. (2010). Classificação dos resultados de enfermagem (NOC). $4^{\mathrm{a}}$ ed. Rio de Janeiro: Elsevier.

- Moro, A., Maurici, A., Valle, J. B., Zaclikevis, V. R., Kleinubing, H., Jr. (2007). Avaliação dos pacientes portadores de lesão por pressão internados em hospital geral. AMB: Revista da Associação Médica Brasileira, 53(4), 300-304.

- Naso, F. C., Pereira, J. S., Beatricci, S. Z., Bianchi, R. G., Dias, A. S., \& Monteiro, M. B. (2011). A classe da NYHA tem relação com a condição funcional e qualidade de vida na insuficiência cardíaca. Fisioterapia e Pesquisa, 18(2), 157-163.

- Nogueira, P. R., Rassi, S., \& Corrêa, K. S. (2010). Perfil epidemiológico, clínico e terapêutico da insuficiência cardíaca em hospital terciário. Arquivos Brasileiros de Cardiologia, 95(3), 392-380. Recuperado de http://www. scielo.br/pdf/abc/v95n3/aop09910.pdf

- Potter, P. A., \& Perry, A. G. (2013). Fundamentos de enfermagem. $8^{\mathrm{a}}$ ed. Rio de Janeiro: Elsevier.
- Risner, P. B. (1986). Diagnosis: analysis and synthesis of data. In: Griftith, K. \& Cristensen, P. J. Nursing Process: application of theories frameworks and models. $2^{\mathrm{a}} \mathrm{ed}$. Saint Louis: Mosby.

- Rogenski, N. M. B., \& Santos, V. L. C. G. (2005). Estudo sobre a incidência de úlceras por pressão em um hospital universitário. Revista latino-americana de enfermagem, 13(4), 474-480. Recuperado de http://www.scielo.br/pdf/ rlae/v13n4/v13n4a03.pdf

- Santos, A. C. S., Santo, F. H. E., Pestana, L., Daher, D. V., \& Santana, R. (2011). Insuficiência cardíaca: estratégias usadas por idosos na busca por qualidade de vida. Revista Brasileira de Enfermagem, 64(5), 857-863.

- Tannure, M. C., \& Gonçalves, A. M. P. (2008). SAE: Sistematização da assistência de enfermagem: guia prático. Rio de Janeiro: Guanabara Koogan.

- Truppel, T. C., Meier M. J., Calixto, R. C., Peruzzo, S. A., \& Crozeta K. (2009). Sistematização da assistência de enfermagem em unidade de terapia intensiva. Revista Brasileira de Enfermagem, 62(2), 221-227. Recuperado de http://www.scielo.br/pdf/reben/v62n2/a08v62n2.pdf

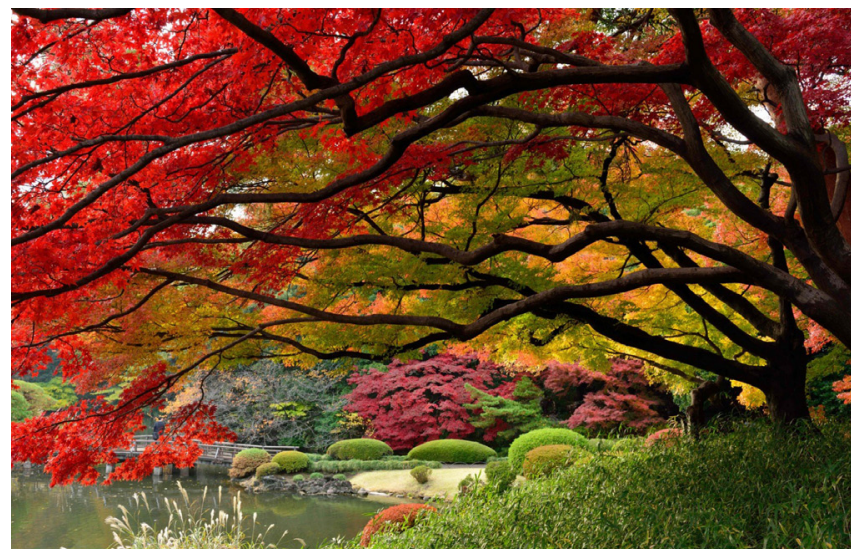

